Historic, archived document

Do not assume content reflects current scientific knowledge, policies, or practices. 


\section{DAHLIA PRICE LIST}

In presenting our 1925 price-list, it is believed you will find listed therein most of the better varieties of Dahlias grown today. These include many of the newest Eastern, Holland and California introductions. Particularly fine is "Midnight," our introduction for 1925. This is a Decorative of the highest class, easily winning first for the best threeyear-old seedling at the Los Angeles Show this year. The color is a deep crimson. overlaid velvety black. Size, eight to ten inches. The foliage is very beautiful, being a dark green and having the appearance of huge holly leaves. The stems are long, stiff, and hold the blooms well above the foliage. Keeping qualities are excellent, and it is a Dahlia that should be in every garden.

\section{POMPONS AND COLLARETTES}

Pompons and Collarettes are becoming more popular each year, and in this list a very complete selection may be found. They are yery profuse bloomers and.will well repay one for planting a few. in additian to the large varieties.

\section{* SEER 6 1931}

Probably the most pleasure in growing Dahlias may be

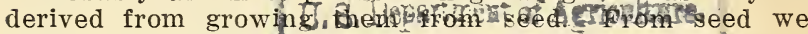
obtain the new varieties, and a seedling seldom produces a flower similar to its parent either in color or formation. Seed should be planted in boxes, kept in a warm, sunny place until four or five inches high, and transplanted to the open ground as soon as the weather permits. They will bloom the first season. We have a very limited amount of seed each year in excess of our requirements, which may be obtained for $\$ 2.00$ per hundred. This seed is hand-selected from the finest varieties grown, and won first for the best seedlings in the two-year, three-year and Pompon classes at the Los Angeles show this year. In ordering seed, please state if you wish Cactus, Hybrid Cactus, Decorative, Peony, Collarette, Pompon or mixed types.

Our tubers are all strong subdivisions taken from fieldgrown clumps, are guaranteed to be true to name, and when shipped to be in growing condition. Any that prove to be otherwise will be cheerfuliy replaced.

Full cultural directions are packed with each order.

PRICES QUOTED are for field-grown subdivisions of strong roots.

FORWARDING.-We ship orders after danger of frost is past.

SUBSTITUTION.-We do not substitute without permission.

TERMS.-Cash with order. Personal checks accepted.

TRANSPORTATION.-We prepay postage on all retail orders.

\section{MULKEY'S DAHLIA GARDENS,} 3863 South Hobart Boulevard,

Member:

Los Angeles, California.

American Dahlia Society.

Dahlia Society of California.

Dahlia Society of Southern California. 
2 Abbr Hougrenard (coile), Lecas lavender, whites (inllar ........................ .50

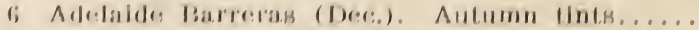

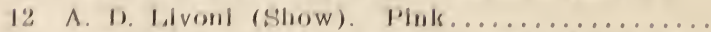

Alrx Waldie (l)ece). Cresum, lavender pencillnges 1.00)

II Amrerea (Doce). Autumn tints........... . 7 Ambassador (Cace). Plols, eream and yellow.. 5.00)

2ti AmI Rorrilat (Slovele). Cismet flowers, purple: lolliages .........................

:Ami Nonin (Coll.). Cerimes rose, whites collar.

I Amun Ras (J)ece), (E)pere and bronze. Very

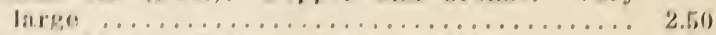

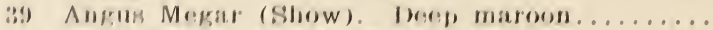

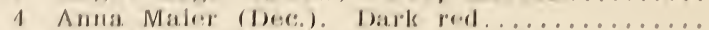

16.2 Apploblomsom (I)ece). Plnk. Imported from

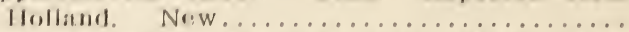

181 Argonne (coll.). Bergindly, wink collar. (RosImtrered.) New..................... .75

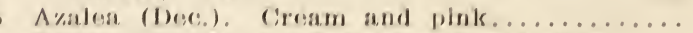

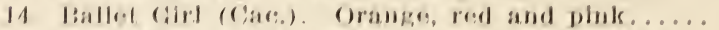

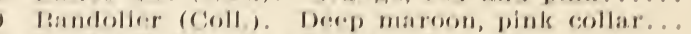

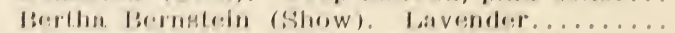
Bertha Horne (H. (ase), (opper and bronze. Britha von Sullnor (II, ('ate.). Pink.......... Isclly Austin (Cace), Hecp, rich pink. Free

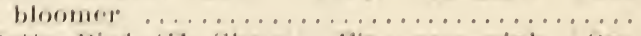

Ix lselly bird (II. Slonw). Nine rosy pink. ()ne

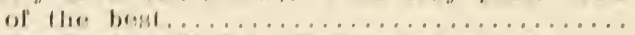
Billice laurke (II, (․). Vellow and eream.....

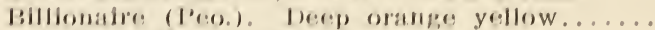

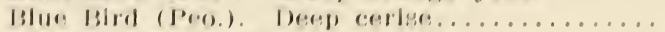

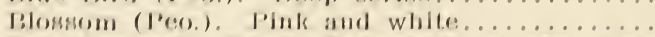

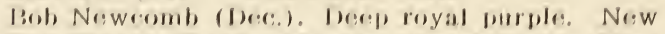
Bob Pleases (I)er:). Maroon, tipecel white. New

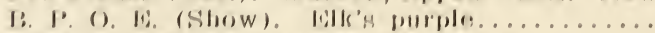

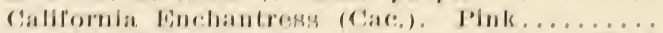

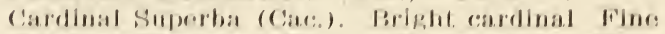
('arolyn Winlgron (J)ece) H'awn pink. Very

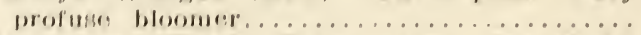

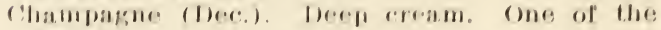
linest

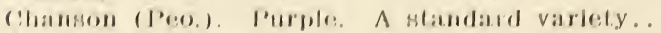
(Elocolate Soldier (cace). Deep maroon...... Cimalerrlla (II, Care.), link and yellow. New

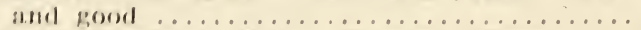

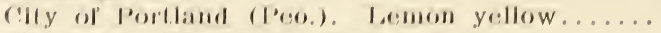

('laremont (H. C:ace), Pink and c:ream.......

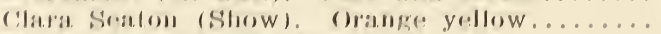
(iontrast (e'ace). Reel, lipped while........

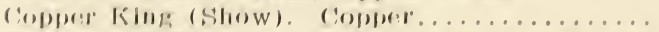
('oumleses 1. Hardegse (coll.). (Eerise, pink and

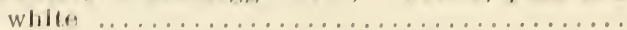

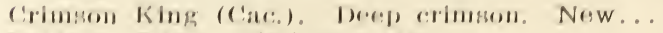
('ro:amo (I)ece). Yallow releam............ (Burlow (Care). Deep lavendar............ loaldy lintler (H. ('ise). Reosy carmine: re:verue, whille...................

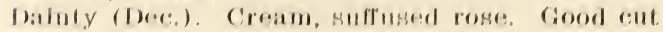
Ilow 11.

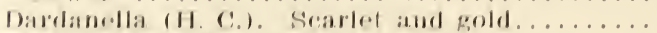

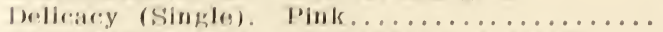
lolla Polles (Dece), lavender and cream....

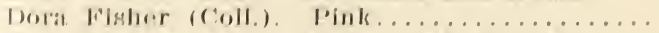
Jorothy de la. Mare (Stanw). Ishasta pink..... Dorolley Inrmbaugh (Cace). Immen: e shell pink 1)1. Peary (Peo.). Dark maroum........... 1)r. Tevile (Deec.). Salmon rose............ 
110) Dundere (H. ('ace), Burnt orange. Very small How erers ......................... . 50

38 biagle ('Thes) (H, Cace), Leleht yellow ....... 41 Earl Williams (Dece). Crimson, lipored whites.

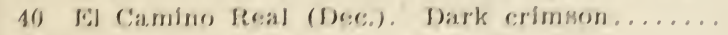

19) Elecetra (Cace). Sulphur yellow ............ 118 Ellizabeth B. Fetler (bee:). Rich purples New

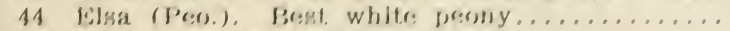

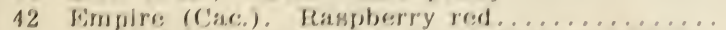

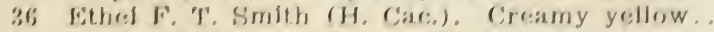

:7 Evelyn Adamson (Jocec.), Fiawn bink.........

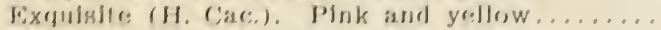

Flreball (Cace), Large crimson. . . . . . . . . 1.50)

Fireburst (f)ere), Reed ............... .35

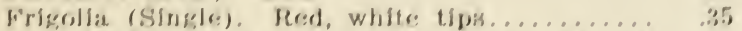

F. W. Fellows (Care), Brieht oranges Ones of the best. ........................

62

(i)

53

6.1

$2: 4$

121

57

56

$8 ; 4$

59

127

128

(ienut. dre Lyon (Coll.). Crimeon, whitse collas . George: Walters (H. ('ace), Sislmon pluk...... Ciec: Whl\% (Ciace), Pink and eream, Very larke:

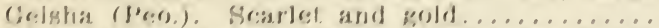

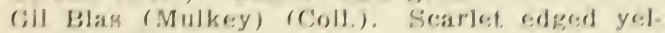
low. Whalle collat. ................ (iladys Mreycrs (I)ece). Yellow............

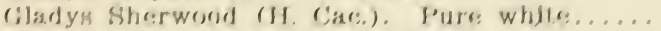

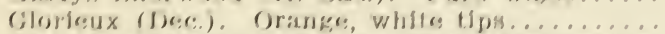
Glory de: Stamhuls (l)rece). Buff. Holland fmprortatfors , ......................

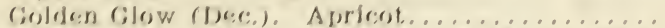
(iolden (joportumity (Shosw). Cisjolen yellows. Nr:w .......................... Colden Rain (ciace), link and yellow .......

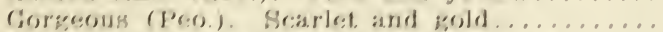
(ioldern Weat. (ciare). Yellow and oranses......

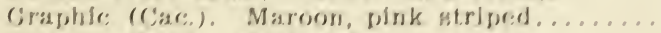

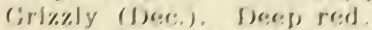

Guardlan (Cace), Crimson,..............

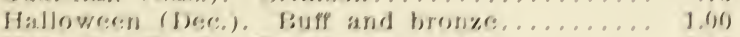

Halvella (J)ere). Briaht rose............ 2nil)

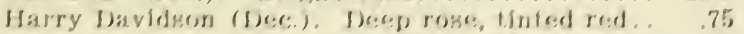

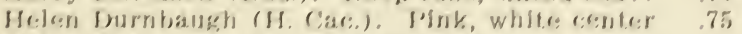

Healen Kelle:r (Heec). Cresimy pink........ .50

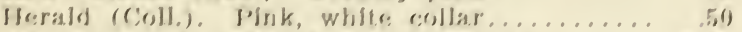

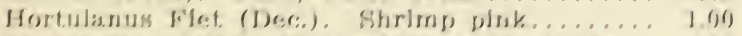

In nosecentla (coll.). Pure: whites, whlle: collest. . 50)

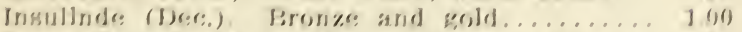

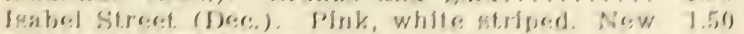

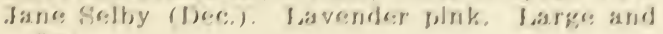
finc:

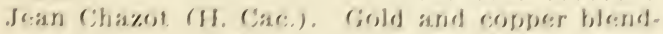
inges

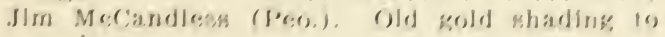
is uriteot.

2.09

Judes: A. B. Parker (J)ee), Fisw with yeilow verncillinises

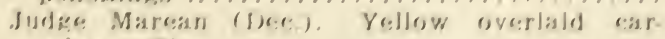
mine Fine.............

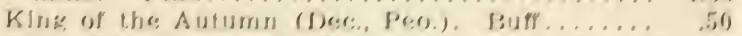

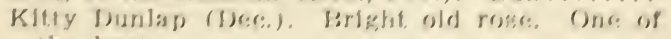

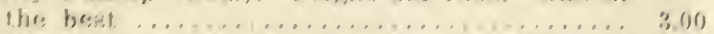

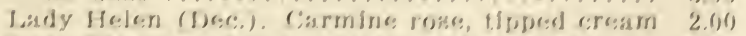

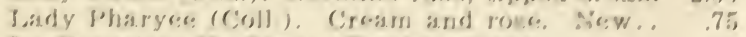

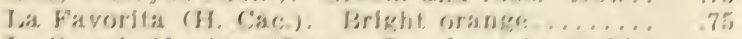

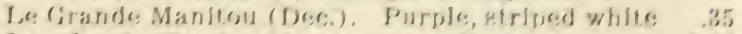

Le:x loord (D)e:e). Maroon, Hssed white...... 200

liberty Bond (f)ece) Buff, yeollow and pirik... 1.06

losletis Velaser) (Ciace), Pure whlle. New and zerod .......................... 500

Mabel V. Talt. (Jocec), Cresm and pirk ..... .75) 
81 Madame Vard (Peo.). Currant red, striped yellow ..................................... 50

88 Magnificent (Cac.). Salmon pink.......... $\quad .75$

214 Marion Cooper (Dec.). Pink............. 1.00

91 Mariposa (H. Cac.). Lavender pink......... 3.50

87 Mary C. Burns (Dec.). Red, reverse yellow... 1.00

79 Mary Purrier (Cac.). Crimson.............. ${ }^{.50}$

169 Maude Adam,s (Show). White, overlaid pink.. $\quad .50$

175 Mephistopheles (Dec.). Ruby red, white mark-

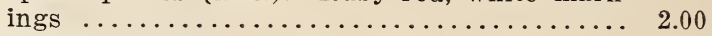

176 Midnight (Mulkey) (Dec.). Very deep red, overlaid a velvety black. Very seldom shows an open center. Has fine, long, stiff stems and fine keeping qualities. This dahlia received first prize for the best three-year-old seedling at the 1924 Los Angeles big show.......... 10.00

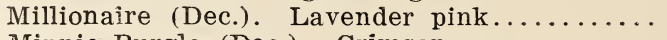

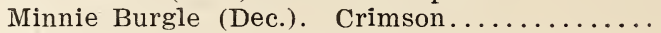
Mons de la Poute (Anemony). Deep red..... Moon Glow (Dec.). Primrose, tipped lilac..... Mr. Crowley (Dec.). Salmon pink, overlaid yel-

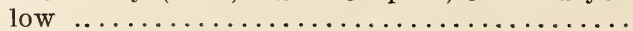
Mrs. A. Stern (H. Cac.). Lavender............ Mrs. F. Bergholz (Cac.). Shell pink. New.... Mrs. C. T. Holcomb (Cac.). Maroon, tipped

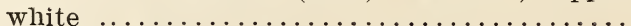
Mrs. Carl Salbach (Dec.). Lavender pink.... Mrs. C. E. Trower (Peo.). Salmon and pink.. Mrs. Edna Spencer (Cac.). Lavender pink... Mrs. I. de ver Warner (Dec.). Deep mauve.

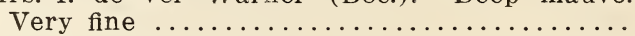
Mrs. Jessie Seal (Peo.). Old rose. One of the best

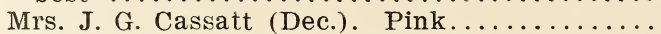
Mrs. Josiah T. Marean (Dec.). Fawn pink, yel-

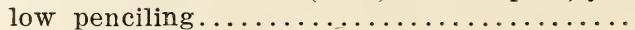

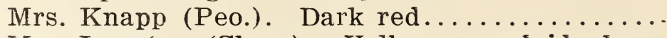
Mrs. Langtry (Show). Yellow, overlaid deep

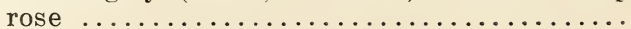
Mrs. O. D. Baldwin (Dec.). Bright magenta... Mrs. Paul Audette (Dec.). Pink and salmon... Mrs. R. R. Strange (Dec.). Deep buff.......... Mrs. W. E. Estes (H. Cac.). Pure white........

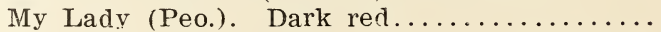
New Moon (H. Cac.). Yellow, occasionally

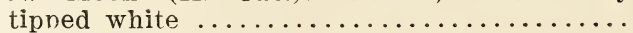
Nebulinguenhort (Cac.). Salmon pink......... Nobilis (Dec.). White, red tipped.............

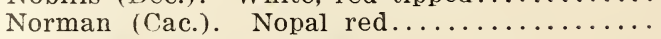

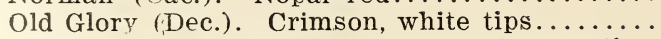
Orange Boven (Dec.). Holland importation.

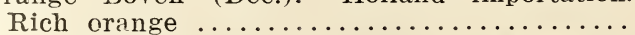
Osam Shudow (Dec.). old rose, yeilow shad-

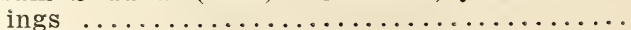

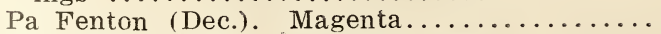
Paul Michael (Dec.). Orange buff............. Pearl Ruggles (Peo.). Bright rose and pink... Pierrot (Cac.). Amber, tipped white........... Pink Triumphant (H. Cac.). Shell Pink. Very

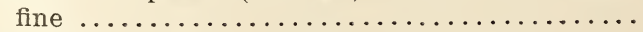
Polar Bear (Dec.). White. Very large.......

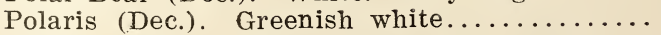
Porthos (Dec.). The so-called blue dahlia.... Pride of California (Dec.). Crimson............ Prima Donna (H. Cac.). Pink and yellow.....

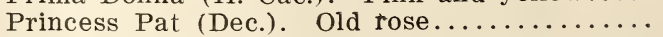
Perry Weidner (Peo.). Bright scarlet. (Reg-

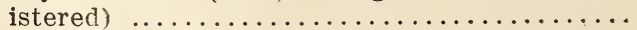

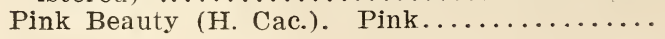

1.00 
You are invited to join

\section{The Dahlia Society of Southern California}

Formed for the purpose of stimulating interest in and promoting the culture and development of the Dahlia.

We publish quarterly Bulletins, free to all members, which contain many instructive articles by experts on Dahlia culture, diseases and remedies, also reports on new varieties, news of the Dahlia shows, etc.

Every member receives a season ticket to our great annual Dahía show, held in September.

Our monthly meetings are always well attended, all members are requested to make use of the question box and open discussions being held on all subjects pertaining to the Dahlia.

Please enter my name as a member of your Society. Enclosed fin $\$ \$ 2.00$ for yearly dues.

Name

Adidress

Town State.

Make all remittances payable to the Society and send to T. F. Schock, Secrstary, 3702 South Gramercy Place, Los Angeles, California. 


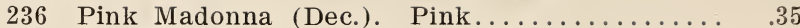

117 Priscilla (Dec.). Pink and white........... .75

222 Quaker (Cac.). Lavender pink and white.... $\quad .50$

241 Quaker City (H. Cac.). Pink................ 75

123 Queen Esther (Peo.). Bright red........... .75

211 Red Insulinde (Dec.). Deep crimson......... 2.50

126 Rheinischer Frohsinn (Cac.). Rose, white tipped $\quad .50$

212 Rising Beauty (H. Cac.). Scarlet and gold.... 1.50

215 Rodman Wanamaker (H. Cac.). Pink, with yel-

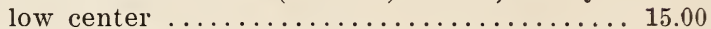

125 Rookwood (Dec.). Cerise rose. Very large and fine ........................ 4.00

124 Rosa Nell (Dec.). Bright rose. Very fine....

216 Scaramouche (Cac.). Nopal red. New and good

133 Schwartzathal (Coll.). Rose, edged white. Fine

135 Scottish Chief (Dec.). Brown, shading to pink

217 Shirley Brown (H. Cac.). Copper and bronze.

137 Shower of Gold (Dec.). Golden yellow........

134 Shudow's Lavender (Dec.). Fine lavender....

131 Silver Medal (Coll.). Rose, edged white; white

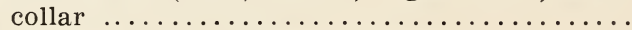

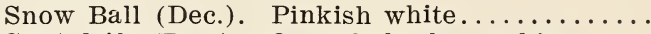

Snowdrift (Dec.). One of the best whites....

Souv. Belle Accuel (Coll.). Orange, yellow

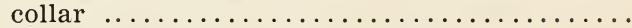

263 Souv. Gust. Doazon (Dec.). Large orange.....

224 Sunkiss (H. Cac.). Pink and cream. New and

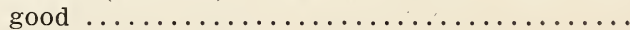

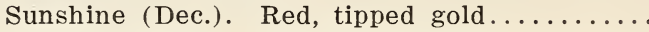

Surprise (Cac.). Orange and yellow.............

Susan G. Tevis (Dec.). Deep lavender. New

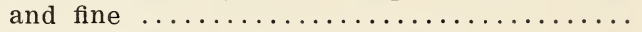

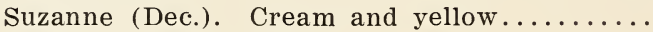

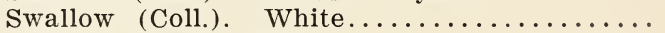

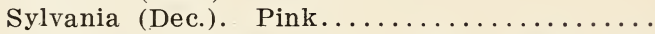

Thelma Jorgensen (Peo.). Gold, orange and

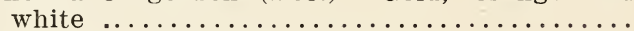

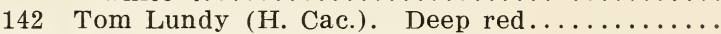

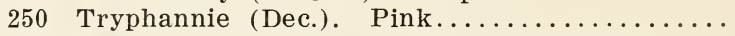

143 Union Jack (Single). White, edged red.......

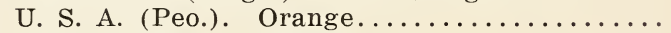

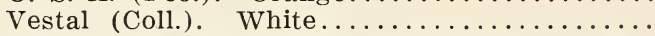

Vicar of Wesparton (Cac.). Yellow and pink.. Virginia May Robinson (Dec.). Yellow.......

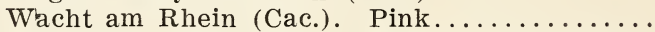
W. D'Arcy Ryan (Dec.). White, tipped purple.

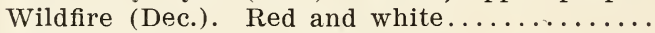
Wm. Welch (Coll.). Yellow, with yellow collar W. W. Rawson (Show). White overlaid mauve Yellow Aster (Dec.). Yellow; petals reflex... Yellow Prince (Dec.). Yellow. Large and per-

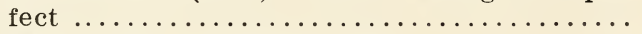

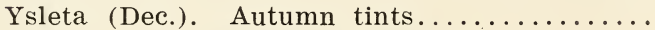

\section{POMPON DAHLIAS}

271

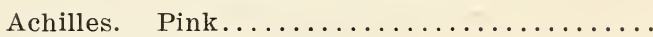

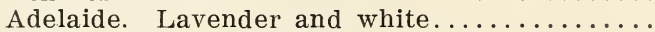

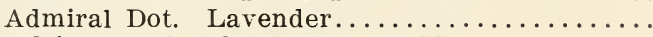

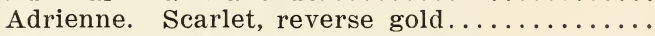
Aimee. One of the smallest and best buffs...

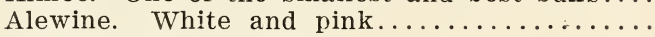

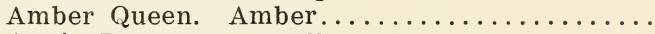
Annie Doncaster. Yellow and mauve........

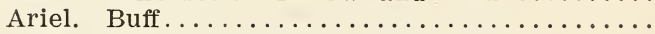
Arthur Kurley. Dark red. Good form.........

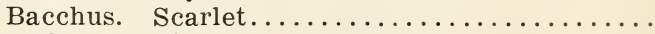

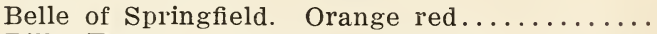
est and most perfect pompon. Three time

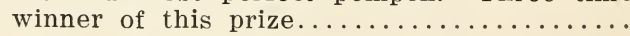


279 Bronze Beauty. Deep buff............ .35

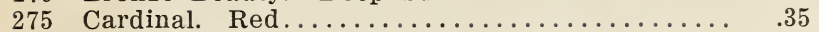

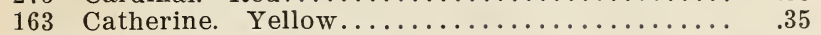

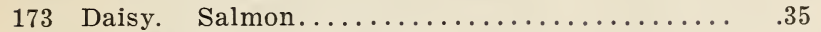

174 Darkest of All. Maroon.......................

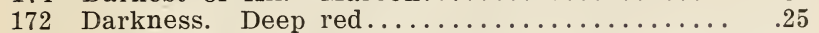

171 Dearest (Mulkey). Bright rose. Fine, but large 1.00

276 Dee Dee. Fine small lavender............. $\quad .75$

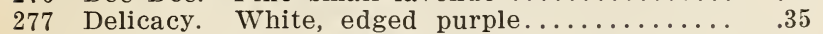

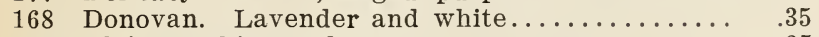

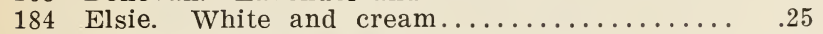

185 Eunice. White, edged mauve............ .35

278 Frau Dr. Knabbe. White, purple edged. Quilled

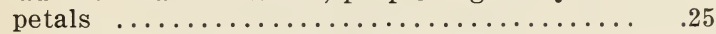

189 Gannymede. Fawn pink. Very fine......... .50

190 Geo. Ireland. Fine lavender............. $\quad .50$

191 Girlie. Beautiful lilac. Fine form.......... .35

192 Glow. Old rose. One of the best........... .50

188 Golden Queen. Yellow. Large, with quilled

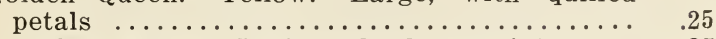

187 Gretchen Heinie. White, edged rose pink..... .35

198. Heckla. One of the best whites.......... .50

279 Helen Cottrell. White and mauve. Fine form. .50

195 Helen Lambert. One of the best deep yellows. $\quad .50$

280 Highland Mary. Pink. Large............... .25

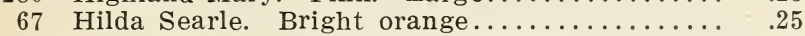

199 Ideal. The best yellow for exhibition........ $\quad .50$

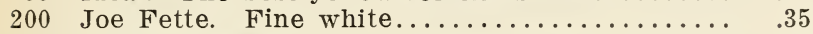

202 Johnnie. Deep maroon. Fine form ............ .50

223 Lassie. Yellow, suffused rose. Perfect form.. .50

281 Little Beauty. Pink, quilled petals.......... .25

225 Little Beeswing. Orange, shaded red......... .35

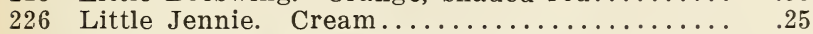

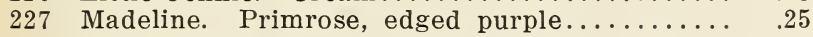

228 Mars. Bright crimson. Fine form........... .35

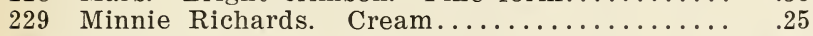

238 Perfection. Good lavender............... .35

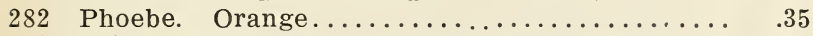

239 Prince Charming. Lavender, edged purple.... .25

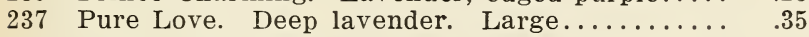

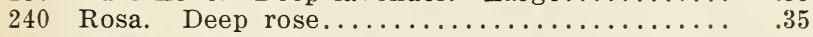

283 Rosea. Lilac. Very fine.................. .50

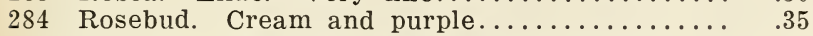

247 San Toy. Pink tipped rose. Fine bloomer.... .35

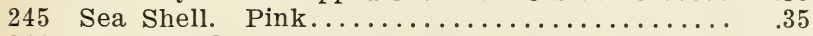

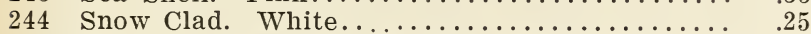

139 Stolz von Berlin. Holland importation. In Europe, is considered one of the finest pink

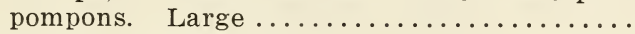

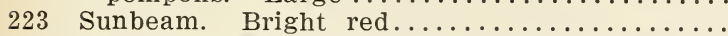

286 Tiny Tim. One of the smallest and best pinks.

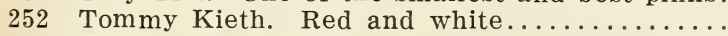

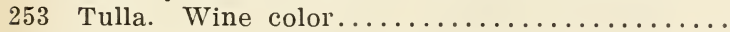

254 Van Esce. Creamy white. Good form........

257 Wee Gracie. White with lavender center. Good

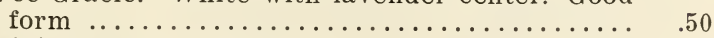

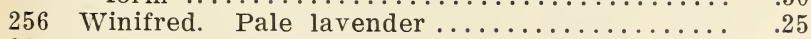

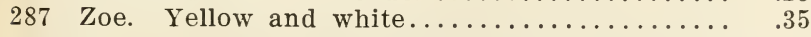

\section{MULKEY'S DAHLIA GARDENS}

3863 S. Hobart Boulevard 


\section{DISCOUNT SHEET}

\section{For Comercial Growers ONLY}

$25 \%$ discount on less than six of one variety, or on mixed orders under $\$ 15.00$.

$331 / 3 \%$ discount on lots of six or more of one variety, or on mixed orders for $\$ 15.00$ or over.

Terms CASH-f. o. b. Los Angeles, Calif. 